\title{
LA CONTRIBUCIÓN DE LA OCUPACIÓN EN LA CONSTRUCCIÓN DE LA CULTURA EN LA FERIA LIBRE DE LA COMUNA DE VALDIVIA
}

\author{
CONTRIBUTION OF OCCUPATION IN THE CONSTRUCTION OF CULTURES IN THE \\ STREET MARKETS OF VALDIVIA CITY
}

\author{
Muñoz B. Danielle' ${ }^{1}$, Pinto V. Romanet ${ }^{2}$, Rosas L. Vanessa ${ }^{3}$, Sánchez G. Macarena ${ }^{4}$, \\ Sánchez Z. Soledad ${ }^{5}$.
}

\begin{abstract}
Resumen
Estudio cualitativo de diseño narrativo. Las técnicas utilizadas fueron, la entrevista en profundidad semi-estructurada y observación participante pasiva. Para ello se realizaron cuatro visitas de campo a la Feria libre Valdivia; se eligieron a cinco actores claves y a su vez, se aplicó una pauta de observación de forma y función del contexto, elaborada por las investigadoras. Los resultados fueron analizados y contrastados con elementos teóricos relacionados.

El objeto de esta investigación guarda relación con la necesidad de abordar la complejidad contextual de esta comunidad bajo la intervención con relevancia cultural desde Terapia Ocupacional, y a su vez responder a la interrogante: ¿Contribuye la ocupación (productiva) a la conformación de cultura en la feria libre Valdivia? ¿Cómo?

Los resultados dan cuenta de la relación de su ocupación productiva, tanto en forma, función y significado, con las características que lo constituyeN como tal, y dan forma a la cultura feriante.
\end{abstract}

\section{Palabras clave:}

Ocupación, cultura, sentido de pertenencia, identidad, estilo de vida y elección ocupacional.

\begin{abstract}
qualitative study with narrative design. Theused techniques were a deep semi-structured interview and passive participant observation. For this, four field visits were performed in Valdivia's Fair, five key players were selected and a context's function and shape observation guideline made by the investigators was applied. The results was analyzed and compared with related theoretical elements. The purpose of this research is related with the need to approach to the contextual complexity of this community under the culturally relevant intervention from Occupational Therapy and in turn answer to the question: Does the occupation (productive occupation) contributes in culture's conformation in the Valdivia's fair? how?.

The results show the relationship of their productive occupations, in form, function and meaning, with features that constitutes it as such and shape the culture in Valdivia's fair.
\end{abstract}

\section{Keywords:}

Occupation, culture, sense of belonging, identity, lifestyle and occupational choice.

1 Terapeuta Ocupacional, Hospital Familiar y Comunitario Gorbea, Carrera 901. Cel:73054789. E-mail: dsmbenner@gmail.com.

2 Licenciada en Ciencias de la Ocupación y Terapeuta Ocupacional, Escuela de Terapia Ocupacional, Facultad de Medicina de la Universidad Austral de Chile. E-mail:romanet.pinto.villalobos@gmail.com.

3 Terapeuta Ocupacional, Hogar San José, Las Condes 13200, Lo Barnechea. Cel: 72145732. E-mail: vanessarosaslabra@gmail.com.

4 Terapeuta Ocupacional, Centro de Salud Mental Comunitario, Germán Hube 735, Osorno. Cel: 56460146. E-mail: macarenasanchezgo@ gmail.com.

5 Terapeuta Ocupacional, Hospital de Rengo y Hospital San Juan de Dios, San Fernando. Cel: 88117852 E-mail: s.sanchezzamorano@gmail.com. 


\section{INTRODUCCIÓN}

Desde tiempos inmemorables, las ferias libres han sido el punto de encuentro público por excelencia, en donde además de abastecer de productos necesarios para la subsistencia de la población, se genera un espacio de intercambio cultural, en el cual se pueden encontrar diferentes expresiones artísticas y fomentar el desarrollo cívico de los pueblos. En nuestro país las ferias libres datan del tiempo de la colonia, en que tales expresiones populares junto a la transmisión oral de los acontecimientos de otras comarcas, lograron reflejar la realidad de las culturas criollas donde se conformaron (Salazar, 2003).

Las Ferias Libres realizan un gran aporte al desarroIlo de la economía nacional abasteciendo al $70 \%$ de la población de productos hortofrutícolas en el país, transando U\$2.600 millones al año; en los sectores de menores ingresos llegan al $90 \%$ de la población. Se contabilizan cerca de 925 ferias libres en todo el territorio nacional, representando a 84.000 emprendimientos familiares, entregando una fuente laboral para al menos 200.000 personas, que trabajan en estos mercados. (Confederación Gremial Nacional de Organizaciones de Ferias Libres, Persas y Afines, 2012).

En Valdivia, la feria se compone de 180 miembros aproximadamente, quienes se organizan por medio de un sindicato conformado por presidente, secretario, tesorero y tres directores. Se establecen en cuatro sectores de la ciudad: Av. Scnheider, Gral. Baquedano, Av. Argentina, sector Corvi y Yañezabala, durante seis días de la semana. Su jornada laboral comienza a las 5:00 AM, aunque las condiciones climáticas sean adversas.

Los feriantes son considerados actores sociales significativos de la idiosincrasia valdiviana y del país en general, que han convivido y experimentado unidos largos periodos de tiempo, creando una identidad cultural propia, a través de experiencias, adquiriendo acuerdos intersubjetivos que han definido una dinámica que los ha diferenciado de cualquier otro conglomerado de individuos. Al respecto Iwama (citado por Iwama, Kapanadze y Simón 2008) nos recuerda que más que la capacidad de hacer, es la necesidad de pertenecer, la que le da sentido de ser a un ser humano.

Existen modelos propios de la práctica con sensibilidad cultural, y como Terapeutas Ocupacionales debemos ser capaces de utilizarlos en pos de comprender las dinámicas que surgen en comunidades específicas.

Es importante enfatizar sobre el ejercicio de la disciplina que: "entendemos que el ser humano se cons- tituye en una compleja red cultural y que la cultura, como un saber colectivo y acumulado en la memoria social generadora de principios y conocimientos, podrá ser liberadora u opresora" (Agostini, Drumond, Zocchi, 2011). El valor de la cultura se puede evidenciar en las ocupaciones que desarrollamos en nuestro hacer, ya que entender el concepto de ocupación y significación es entregarle valor a lo que en lo cotidiano realizamos.

Iwama (2008) señala: "La ciencia de la ocupación no se puede convertir en una entidad cultural en sí misma que se desarrolle bajo los parámetros culturales del paradigma en donde se está desarrollando. Debe ser capaz de validar las realidades y contextos de personas que están excluidas del paradigma de investigación predominante". Guajardo (2010) afirma: "Nuestra existencia -Terapeutas Ocupacionales-, no deviene de un orden natural o de una condición intrínseca, por el contrario, debemos nuestra existencia a determinados problemas sociales, expresados en ámbitos como salud, educación, trabajo, justicia y/o de protección social".

Bajo un paradigma social en Terapia Ocupacional (T.O.) y desde la Ciencia de la Ocupación, la presente investigación, realizada en el marco de trabajo del seminario de título por estudiantes de quinto año de la carrera Terapia Ocupacional de la Universidad Austral de Chile, tiene como objetivo analizar la contribución de la ocupación en la construcción de cultura en la Feria Libre en Valdivia. A continuación, se describirán aspectos generales del contexto socio-histórico de la formación de ferias libres a nivel nacional y local, elementos conducentes a conceptualización de cultura, desde la T.O. presentes en el grupo de estudio; y aspectos generales de la historia de vida de actores clave de la feria libre Valdivia.

\section{MÉTOdo}

La presente investigación, se inicia en el mes de agosto 2012 hasta n oviembre del 2013, durante su desarrollo se realizarán seis sesiones de aproximación, trabajo en campo, recolección de datos, discusión y análisis de los resultados.

Según los objetivos que persigue la investigación, se trabajará en base a la metodología cualitativa, pues ésta nos permite "el entendimiento del fenómeno en todas sus dimensiones, internas y externas, pasadas y presentes" (Baptista, Fernández y Hernández, 2006). 
Las técnicas consideradas para este fin, son la entrevista en profundidad semi-estructurada y la observación participante pasiva. Ambas realizadas en el contexto de la feria.

En los cuatro primeros acercamientos a la comunidad, con el objetivo de compartir sus usos, costumbres, estilo y modalidades de vida, se utiliza la observación participante, en las cual se evidencia de qué forma se lleva a cabo la feria, es decir, cuáles son las actividades que se incluyen, los horarios que implica desempeñarse en ella, el vestuario que utilizan, el lenguaje, entre otros. Además en aquellas instancias, se establece el vínculo con representantes de la directiva y otros trabajadores sugeridos por los mismos.

En base a lo propuesto y a los objetos planteados, la selección de sujetos de la muestra de investigación está compuesta por cinco miembros de la comunidad de la "Feria Libre" de la ciudad de Valdivia, para lo cual se consideró a dos personas bajo los criterios de "Excelencia o búsqueda del gran hombre" y tres personas bajo el criterio de "Normalidad".

A cada uno de ellos se le solicitará su consentimiento informado para participar de la investigación a través de un documento elaborado por el grupo de autoras y aprobado por el comité de ética de investigación del Servicio de Salud Valdivia. En él se detalla el carácter académico de la investigación con miras a una publicación a futuro, se especifica la confidencialidad y la inexistencia de retribución económica por la participación en él.

Se prosigue con la aplicación de la entrevista semiestructurada, con el objetivo de comprender las perspectivas que tienen los informantes respecto de sus vidas en un encuentro cara a cara entre el investigador y el informante. Ésta consta de una pauta con 20 preguntas aproximadamente, que ordena aspectos clave respecto antecedentes generales de los entrevistados, funcionamiento detallado de la feria, elementos socio-históricos de la formación de éstas, aspectos positivos y negativos del trabajo como feriantes, sentimientos evocados en relación a la ocupación de desempeñarse en dicho lugar, entre otros. Dependiendo de la extensión de las respuestas entregadas por los participantes, se da la oportunidad de contestar la pauta en 1 o 2 tandas. Se grabarán las entrevistas para posteriormente digitalizarlas y ser analizadas por el grupo de investigadoras. El análisis de los resultados se realizará a través de la comparación del análisis obtenido en contraste con la teoría.

En esta investigación se aproximará al lector a la definición de cultura, desde aportes de la antropología y la Terapia Ocupacional. En relación a la primera, Marvin Harris (2000) entiende cultura como "El conjunto de tradiciones y estilos de vida socialmente adquiridos de los miembros de una sociedad en donde influye en sus modos pauteados de pensar, sentir y actuar". Sir Edward Burnett Tylor en 1871 (citado por Harris 2000) agrega: "La cultura... es ese todo complejo que comprende conocimientos, creencias, arte, moral, derecho, costumbres y cualesquiera otras capacidades y hábitos adquiridos por el hombre en tanto que miembro de la sociedad..." Desde Terapia Ocupacional se suma, "la cultura representa mucho más que las características materiales que distinguen a unas personas de otras. Es un fenómeno dinámico, una interacción compleja de significados que representa y conforma la vida individual y colectiva de las personas" (Iwama y Simó, 2008).

Finalmente, se abordarán conceptos propios de la Terapia Ocupacional. Al respecto, Charles Christiansen define ocupación como: "el medio principal a través del cual las personas se desarrollan y expresan sus ideas personales" (Iwama y Simó, 2008). El autor añade que "la ocupación humana es el diálogo entre la persona (grupo o comunidad) y el medio" (Simó, 2012), agregando el componente contextual.

El sentido de pertenencia, se entenderá como: "el sentirse parte de un lugar, sentir que el lugar donde se está, es propio (...) ya que si una persona se siente parte de un lugar, lo siente propio, actúa en él, haciendo cambios, tomando responsabilidades frente a él y teniendo derechos también". Por su parte, la identidad: "serán aquellas raíces que dan un sustento y sentido de pertenencia (Tappan, 1992)"; éstas se generan en las interacciones sociales cotidianas que mantienen los sujetos entre sí, a través de las cuales van delimitando lo propio contra lo ajeno (Piqueras, 1996).

\section{Resultados}

\section{Elementos del contexto socio-histórico de la formación de ferias libres a nivel nacional y local, identificados por los entrevistados}

Actualmente, la feria abarca diversos sectores de Valdivia con carácter itinerante. Cuenta con 68 puestos; de ellos 29 de los propietarios son hombres y 39 mujeres. Está compuesta por 180 feriantes, observándose dos a cuatro personas por puesto. 
Cuando se les solicita a los entrevistados, información respecto a la historia local y nacional de la feria, se rescata que existen nociones comunes sobre su conformación e historia a nivel local. Sin embargo, se desconoce en su mayoría la organización a nivel nacional, excepto cuando se trata de trabajadores participantes de la directiva, que poseen gran nivel de conocimiento respecto a la organización interna de la feria: existe conocimiento de la organización del sindicato y de los miembros que la componen, reflejándose en el discurso de cada uno de ellos. Esta organización se compone por el presidente, un secretario/a, un tesorero/a y dos directores.

\section{Antecedentes generales de los entrevistados y sus respectivos puestos de trabajo}

Los feriantes son un grupo heterogéneo, que se distribuyen aleatoriamente. Su comportamiento guarda relación con el objetivo de la actividad productiva, que en este caso, es la comercialización de productos. En cuanto al grupo específico de entrevistados, la edad oscila entre los 45 y 65 años, en su mayoría casados/as, quienes comparten labores dentro de la feria.

En relación al ambiente físico de la feria, los puestos de los trabajadores se disponen unos junto a otros en toda la extensión de las calles principales, tres a seis cuadras aproximadamente, designadas según ordenanza municipal. Se compone de puestos con dimensiones de tres por tres metros, elaborados con barras de fierro y toldos impermeables. Cada feriante cuenta con su propio montaje que incluye materiales como pesas, bolsas y diarios. Sumado a esto, el trabajador es responsable de contar con sus recursos materiales (silla descanso, calefacción, comestibles, etc.).

En relación a la vestimenta no existe consenso sobre el uso de uniforme, sin embargo, la mayoría de las y los trabajadores utilizan ropa cómoda, priorizando prendas que proporcionan abrigo y protección térmica. No existen espacios comunes de descanso, alimentación o recreación, tampoco cuentan con infraestructura que les proteja de condiciones climáticas extremas, características de la zona sur.

En cuanto a las funciones que se ejercen en la feria, éstas son bastante variadas; principalmente destaca la de vendedor y la de voceador. Las tareas cotidianas que realizan las personas, básicamente incluyen: instalar el puesto (fierros, toldos, cajones), ordenar los productos que serán vendidos, atender al público y desinstalar el local. Las actividades que realizan los participantes son múltiples, pero es interesante conocer que los tipos de productos con los que trabajan varían de acuerdo a los recursos con los que cuentan, por ejemplo, existen feriantes que optan por vender quesos, pues no requieren de un puesto con grandes estructuras. A esto se suma el aspecto relacionado al transporte, ya que se requiere de un vehículo para transportar las estructuras, mercaderías, etc. y a su vez, es necesario contar con una persona que pueda ensamblar los puestos de trabajo.

La interacción con el público es permanente, los vendedores diariamente invitan a comprar su mercadería a través de cánticos propios en donde se resaltan las mejores características de los productos. Al momento de preguntar por algún producto se entabla una conversación amena, en donde existe un intercambio de experiencias. Los vendedores tienen un trato amable con todos los clientes, ya que existe la posibilidad de que este vuelva y se convierta en un "casero" (cliente frecuente).

Se les solicita a los participantes que expresen aspectos positivos de su trabajo. Al respecto señalan, la posibilidad que este lugar les brinda de compartir con amigos, generar vínculos de apoyo, conocer gente nueva, entre otros aspectos. Además, mencionan los beneficios económicos implicados en ella. En cuanto a aspectos negativos, manifiestan falta de apoyo por parte de las autoridades, políticas públicas que los amparen, condiciones laborales precarias, pequeños puestos para instalarse a trabajar y la falta de participación comunitaria por parte de los mismos trabajadores. Los feriantes señalan que los cambios que realizarían a la feria, se relacionan con el traslado de ésta a un lugar que les proporcione mayor estabilidad.

\section{Las causas y consecuencias de la elección ocupacional del "ser feriantes" a través de la historia de vida de los entrevistados}

Se estima, según los datos entregados, que los participantes han permanecido en el lugar entre doce y treinta años. En algunos casos, su llegada se vincula directamente con familiares ya establecidos en la feria libre; en otros, como consecuencia del desempleo y formación estudiantil incompleta u oficio ligado al sector rural de procedencia. Frente a la interrogante de la existencia de otros familiares ligados al rubro, se puede mencionar que los feriantes mantienen por lo general a algún miembro de su familia que participa en apoyo o trabaja en el mismo. 
En lo que respecta a la historia laboral de los entrevistados, se rescata que también han trabajado en otras áreas que no tienen similitudes con lo desempeñado en la feria libre, sin embargo, refieren que al haber elegido esta ocupación, se sienten comprometidos, integrándola dentro de su historia. Esto demuestra la permanencia en esta, siendo parte de su vida y rutina. En otro aspecto, se evidencia la existencia de participantes que han llegado a desempeñarse en este contexto por razones más bien de carácter económicos y no necesariamente por tradición.

Se refleja la importancia de la influencia familiar en la elección de la ocupación (trabajar en la feria), siendo esto generacional. No obstante, algunos participantes sostienen que sus hijos optaron por explorar otros oficios. En la gran mayoría de los casos, miembros de la familia comparten los quehaceres del trabajo en la feria.

\section{Elementos desde la ciencias de la ocupación inmersos en el discurso de los entrevistados}

En relación a la rutina diaria de los entrevistados, ésta se estructura según las necesidades y demandas de las ocupaciones relacionadas a su rol productivo dentro de la feria. La dinámica de trabajo comprende: el comienzo del día, en promedio es a las seis de la mañana llegando a las siete de la tarde a su hogar para realizar quehaceres. Las comidas, como el desayuno y el almuerzo, se dan dentro del espacio de trabajo, compartiendo junto a sus compañeros de labores, utilizándolo como instancia de participación social.

Durante la entrevista, se les solicita que se autodefinan, lo cual realizan través de características propias, mencionando como principal aspecto ser "trabajadores" (feriantes), "sacrificados", "buenos compañeros", "personas alegres". Así mismo, se considera relevante indicar la importancia de los rasgos personales y el grado de simpatía para atender a los clientes. En algunas respuestas, se mencionan relatos como: "Soy un hombre trabajador, a mi edad trabajo y me gustaría seguir trabajando aquí en la feria" y otros como "amable con la gente, condescendiente, pero amable para poder vender".

En este mismo sentido, los entrevistados señalan que permanecen trabajando en la feria libre, pues es la solución económica cuando los requerimientos de trabajos formales no se adecuan a sus necesidades o a su realidad (jubilados, sin acceso a educación, entre otros).

El significado que le otorgan las personas que desempeñan un rol laboral en la feria libre, se orienta principalmente al nivel de satisfacción que les provoca desempeñar dicho rol. Además, como recientemente se menciona, este trabajo es considerado como un medio para generar ingresos que sustenten económicamente sus distintas y variadas necesidades.

Como elemento central se repite el concepto de "familia", pues ellos refieren que la feria libre se ha constituido para ellos como tal. A pesar de lo anterior, recalcan lo "sufrido" que resulta su trabajo, mas estas condiciones laborales no serían motivo para abandonar las responsabilidades que han adquirido. No aparece como elemento de pertenencia el territorio, pues, o no lo mencionan, o refieren deseos de irse hacia otro lugar con mejores opciones laborales. Sobre lo anterior, también se repite el factor temporal como una importante variable a considerar, explicando que el prolongado periodo que llevan desempeñándose en dicho lugar, ha impactado en sentirse parte de él.

Relacionado al grado de satisfacción de trabajar en la feria libre, los participantes señalan que disfrutan participando de esta actividad, aunque algunos manifiestan que esto va más allá de la feria en sí, dicen que tiene relación con el sentirse productivos.

En relación a la importancia que los mismos miembros de la feria libre le otorgan a ésta, cabe destacar que la consideran una fuente de trabajo que les da un ingreso económico, además proporciona productos frescos a precios accesibles, generando, por otra parte, la opción de compartir e intercambiar experiencias, desarrollando un vínculo entre el feriante y el "casero".

\section{DisCUSIÓN}

\section{Elementos del contexto socio-histórico de la formación de ferias libres a nivel nacional y local identificados por los entrevistados}

En relación a los elementos del contexto socio-histórico de la formación de ferias libres, se obtuvieron importantes datos que abren el análisis sobre fenómenos sociales que han permitido la conformación y consolidación de la Feria libre como patrimonio cultural de la Región de Los Ríos.

Al indagar respecto a la génesis de la feria, confluyen en que nace hace cuarenta y dos años, en donde feriantes se atrevieron a establecerse en el puente Pedro de Valdivia, con puestos que exhibían frutas y verduras 
de la estación para ser comercializadas a la población que llegaba hasta allí para abastecerse; durante este periodo no era itinerante. Posteriormente, luego de su legalización en 1968, la feria se toma los espacios públicos y logra llegar a toda la población valdiviana realizando un trazado itinerario.

Esto nos demuestra la falta de conciencia sobre otros elementos socio-históricos, principalmente aquellos relacionados a la conformación a nivel nacional, pues conocen, producto de la transmisión oral de sucesos, únicamente cómo han sucedido los hechos a nivel local. Según la información recabada por ellos, son más importantes las relaciones que se establecen durante los procesos que son propios de cada feria, por lo que no muestran interés por conocer información acerca de la conformación de ferias libres a nivel macro, ya que no las sienten propias.

\section{Antecedentes generales de los entrevistados $y$ sus respectivos puestos de trabajo}

De acuerdo a la información recabada de la aplicación de las entrevistas individuales, se puede mencionar que los feriantes componen un grupo cuyos miembros poseen características similares, como ser parte de los estratos vulnerables de la sociedad, quienes presentan elementos comunes: escolaridad incompleta, iniciación laboral a temprana edad debido a necesidades de supervivencia, actividad agrícola vinculada al territorio rural de pertenencia o heredada desde sus padres, baja tolerancia a trabajos con extensas jornadas; todos ellos dan cuenta del ser feriantes como una oportunidad para obtener ingresos para el bienestar económico familiar.

Otro factor característico que se evidencia, es la clara influencia de esta elección en sus familiares, dando lugar a sus decisiones en el ámbito laboral. Existen otros aspectos identificados, como el lenguaje y las estrategias que adquieren para favorecer la venta de sus productos como los cánticos y frases características, usadas como técnica para atraer al cliente. La elección de esta actividad que se transmite vía transgeneracional, guarda directa relación con la definición de materialismo cultural que propone Marvin Harris (2000), que define que los individuos se configuran sobre una infraestructura, siendo esta la estructura más básica, que asegura la subsistencia biológica de los individuos del grupo, la sustentabilidad en el tiempo y el espacio del grupo.
Con respecto al espacio físico, todos los miembros de esta comunidad cuentan con un puesto con aspectos similares como las dimensiones, los materiales y objetos para construir el mismo. Todos ellos se configuran para estructurar en un ambiente previamente determinado que les permite llevar a cabo el objetivo en común (ámbito comercial).

Por otro lado, se identifican aspectos particulares de las personas que trabajan en la feria en relación a su estilo de vida, estructurando sus rutinas diarias según las tareas necesarias para mantener la actividad laboral. Lo anterior se fundamenta según la siguiente definición de cultura: "el conjunto de tradiciones y estilos de vida socialmente adquiridos de los miembros de una sociedad en donde influye en sus modos pauteados de pensar, sentir y actuar" (Marvin Harris, 2000).

En cuanto al reconocimiento de aspectos positivos del trabajo los denominadores comunes fueron: el trabajo en la feria les brinda un lugar para compartir con amigos, poder generar vínculos de apoyo y una estabilidad económica para sus familias. Esto se puede explicar desde una investigación realizada por el historiador Gabriel Salazar, quien señala que las ferias libres han sido trabajadas cuando menos por dos generaciones de feriantes, en la que los hijos de los fundadores también han sido feriantes en donde los comerciantes de esta pequeña "lonja de abasto" han trabajado en ellas como familias, es decir con un sentido fuertemente comunitario. Incluso la asociatividad -tan social como gremialentre los feriantes tiene el sello comunitario propio de los núcleos familiares (Salazar, 2003).

Respecto a aspectos considerados negativos, manifiestan falta de apoyo por parte de las autoridades, falta de políticas públicas que los amparen, condiciones laborales precarias, entre otras. De ello podemos señalar que las ferias libres representan de algún modo la recuperación parcial de la soberanía del pueblo, ya que es un espacio en donde se pueden llevar a cabo transacciones fuera de los parámetros del sistema neoliberal; es una instancia puramente ciudadana y democrática en donde se brinda la libertad de poder abastecernos de artículos de primera necesidad que no estén normados desde los parámetros del mercado estatista que nos imponen, de alguna manera podemos elegir y acceder desde otro mecanismo. Es por ello que históricamente se ha luchado para la extinción de las ferias libres, sólo en este último tiempo se ha cambiado la visión que se tenía de ellos, pues ahora se le otorga más importancia a la generación de microempresas para disminuir la tasa de desempleo y al aporte que pueda hacer la 
población vulnerable a través de una actividad productiva, para el mantenimiento de la economía nacional.

En la actualidad, existe en el parlamento el proyecto de Ley sobre ferias libres que busca un marco regulatorio para su desarrollo, consolidación y modernización. Nace como una necesidad desde una feria libre de una comuna de la Región Metropolitana para fiscalizar la situación de las mismas y levantar una organización nacional. Pero a pesar de esto, la población de feriantes de Valdivia no tiene información sobre esta organización debido a que esta iniciativa aún se encuentra congelada en la Cámara de Diputados y generalmente esta información queda centralizada en las principales regiones y no se difunde a través de los canales adecuados a los feriantes de regiones más apartadas del país.

\section{Las causas y consecuencias de la elección ocupacional del "ser feriantes" a través de la historia de vida de los entrevistados}

En este nuevo aspecto, cabe destacar que existen múltiples factores que han llevado a la incorporación de los participantes a esta actividad, aunque la causa principal de la elección ocupacional del "ser feriante" se relaciona con un factor ligado, principalmente, a la historia de vida, más concretamente con la participación de algún miembro de la familia en ello, pudiendo denominar a esto de "carácter generacional", en donde los hijos de padres feriantes siguen la tradición y continúan en aquello, inculcando de igual forma a futuras generaciones. En algunas ocasiones no es familia directa, pero sí hay un nexo (conocido, familia de esposa/o, etc.) quien propicia la incorporación a la misma.

Marvin Harris (2000) explicaría este fenómeno de transmisión de cultura, a través del concepto enda culturación, que detalla que la "transmisión de rasgos culturales es por vía generacional, por medio de la experiencia de aprendizaje parcialmente consciente $y / o$ inconsciente en donde la generación de más edad incita, induce y obliga a la generación más joven a adoptar los modos de pensar y de comportamiento tradicionales".

Además, la participación de la familia parece ser una característica principal en el comercio minorista y popular como las ferias libres, ya que esto de alguna forma reduce los costos de operación al mínimo y es utilizado como una estrategia de sobrevivencia exitosa a la cual se recurre cada vez que el trabajo asalariado no responde ni a las necesidades ni a las expectativas.
En cuanto a las consecuencias a través de la historia de vida, éstas básicamente se relacionan con formación de identidad, la satisfacción de trabajar en dicho lugar, referida por uno de lo entrevistados: "me encuentro feliz aquí, más que en mi casa"; el sentimiento de pertenencia, los lazos creados con aquellas personas que trabajan en el rubro: "acá somos todos unidos, se enferma alguien y todos los ayudamos"; la organización de rutinas en torno al desempeño de la actividad productiva, entre otras, que serán detalladas en el eje siguiente. Si bien es cierto, algunos de los entrevistados se han desempeñado en otras labores, siempre han vuelto a la feria, pues "de ese lugar son parte".

En base a lo anterior, se puede comprobar que el sentido de pertenencia, la cotidianidad, es una fuente de reafirmación en escenarios de construcción de valores y momentos vivenciales individuales y colectivos. (García y Montero, 2005). Así también como Anthony Cohen (1986) sostiene que el sentido de pertenencia a un grupo o una comunidad, es decir, lo que significa "ser miembro de..." es evocado constantemente por cualquier medio, ya sea la utilización del lenguaje, la destreza hacia ciertos oficios, el conocimiento de la ecología, de la genealogía, etc. Todo eso tiene sentido y significa algo para ellos, que no significa para los otros. Plantea que para comprender un fenómeno cultural debemos capturar su sentido experimental, vivencial, es decir, lo que "se siente" pertenecer a esa cultura. Todos los cambios y procesos se experimentan de diferentes formas bajo circunstancias distintas. Los significados difieren, las respuestas de los individuos no son mecánicas y pertenecen al campo de lo "simbólico".

\section{Elementos desde la ciencia de la ocupación inmersos en el discurso de los entrevistados}

Al unificar y razonar elementos de la ciencia de la ocupación, en cuanto a la complejidad de esta misma en la vida de los integrantes de la feria libre, articulando de manera sistemática el conocimiento de la forma, función y significado, inmersos en el discurso de los entrevistados, se menciona que:

Según la forma, entendida por Larson, E.; Wood, W.; Clark F,(2005) como: elementos físicos y contextos socioculturales preexistentes que producen, guían o estructuran el desempeño humano, en este contexto, la ocupación productiva llevada a cabo, implica participar en un ambiente con condiciones particulares que la caracterizan como tal. Dentro de ellas, la ubicación 
de puestos laborales creados de forma artesanal, instalados en distintos espacios públicos, le brindan un carácter itinerante. A su vez las situaciones laborales adversas relacionadas con la escasa protección frente a las condiciones climáticas características de la zona, propician un estilo laboral específico que involucra acciones de adaptación a las demandas del medio.

Segundo, como menciona el autor Willard (2005), el gran poder de la ocupación como un instrumento de adaptación, es un medio vital por el cual los seres vivos se adaptan a los ambientes para sobrevivir y desarrollar su potencial, explica el desarrollo de diversos mecanismos de adaptación de los feriantes, a las adversas necesidades del medio, en relación a sus posibilidades y/ou oportunidades. Dentro de ellos, para poder adaptarse a las condiciones socio-laborales, los hábitos de rutina juegan un rol elemental, ya que la jornada laboral es extensa, sin horarios específicos para alimentación, higiene menor y/o descanso. Por otro lado, las actividades ligadas al rol de padre/madre, también han sido incluidas dentro de esta rutina. Se suman las actividades de tiempo libre y esparcimiento familiar. Todo ello dentro del mismo espacio y tiempo cotidiano.

A su vez, el desempeñar esta labor implica estilos de relacionarse y de comunicarse específicos, que los distinguen del común. Los códigos comunicativos difieren de los utilizados por el resto de las personas, éstos contemplan neologismos y estilos verbales y paraverbales propios, transmitidos entre generaciones, sello distintivo de la idiosincrasia del feriante. Crepeau (citado por Willard, H.; Spackman, C.; Crepeau, E.; Cohn, E:, Shell, B., 2005) señala que los miembros de la cultura dan nombre a las ocupaciones, por lo tanto el lenguaje cotidiano las designa fácilmente, porque están compuestas por actividades habituales más que guiadas por la idiosincrasia, pues las palabras significan cosas que las personas en la cultura realizan repetidas veces.

Los estilos de relacionarse con el público y/o clientes determinan una forma específica, pues están basados en un trato acogedor, cordial, informal, personalizado y nutritivo con constante intercambio de experiencias, además en ello se otorgan instancias de un compartir basado en el diálogo afectuoso, configurándose un contexto familiar que invita a compartir.

Desde la función ocupacional, la ocupación productiva llevada a cabo en la feria libre, posee un nexo entre el desempeño de la ocupación y el estado de bienestar que esta conlleva, como medio de adaptación del individuo en su ambiente.
Dentro de los objetivos y motivos por los cuales los individuos desarrollan y participan en esta ocupación, que permiten suplir sus necesidades e involucrarse en actividades de su interés, podemos encontrar en primera instancia el sentimiento de pertenencia al lugar, espacio en el cual han permanecido por largos periodos de tiempo. En este ambiente laboral los individuos se sienten y se auto-refieren como elemento importante en este sistema, siendo influyente y contribuyente en la dinámica general de este, identificándose con sus costumbres, rituales, tradiciones y acciones.

Por otra parte, esta ocupación es considerada como fuente de bienestar y principal ingreso económico, en donde los individuos han aprendido y desempeñado su trabajo para suplir necesidades atingentes en la contingencia de la vida humana, en donde han logrado alcanzar un grado de especificidad en cada uno de sus roles, mediante la experiencia alcanzada en los años de trabajo. A su vez las escasas oportunidades otorgadas por el contexto sociopolítico en el ámbito laboral conllevan a que los trabajos formales no se ajustan a sus realidades y/o necesidades. Mientras que la dificultad en el acceso de la educación y a las políticas públicas contribuyen aún más a la búsqueda de supervivencia y adaptación económica mediante vías informales y desprotegidas.

En el significado, definido por Willard H.; Spackman, C.; Crepeau, E.; Cohn, E; Shell, B. (2005), como la experiencia subjetiva de la participación en ocupaciones, el ser feriante se ha relacionado principalmente al sentido de pertenencia a una red familiar-social. Es común en sus discursos identificar la relevancia que se le atribuye a la dinámica relacional caracterizada como un apoyo permanente, afectuoso y solidario. Motivo por el que varios integrantes permanecen trabajando aun cuando económicamente no lo necesitan, tal es el caso de adultos mayores o adultos jóvenes que han tenido la opción de cambiar de rubro o de trabajo y han señalado volver por no adaptarse al contexto despersonalizado y normalizado.

Por otra parte, se rescata que este sentido de pertenencia, entendido como "el sentirse parte de un lugar, sentir que el lugar donde se está, es propio" (Tappan, 1992), se debe principalmente a las características propias de este oficio, donde no existen horarios ni jerarquía y donde se establecen vínculos afectivos de apoyo mutuo, compartiendo desayunos, almuerzos, dificultades familiares, por nombrar algunas. Según Redon (2010), el sentido de pertenencia resulta esencial 
al hablar de cohesión y formación ciudadana, ya que al sentirse parte, el sujeto actúa en dicho lugar", lo que explicaría desde otra arista la incorporación de miembros de la familia, pues la feria se vuelve su centro de actuación y por tanto los obliga a utilizarlo para compartir instancias familiares.

Asimismo, el desempeñar el rol de feriante significa para ellos su fuente principal de ingresos. Pero es más que eso, según se rescata en los resultados, es considerado como una labor sin control externo, libre, según señalan, que les permite desempeñarse en variadas ocupaciones y roles a la vez, sobre todo en aspectos relacionados con maternidad.

El compartir y el ambiente alegre y particular de la feria es altamente valorado por sus miembros. Se rescata un sentido de placer y satisfacción al participar en ella junto a un sentido de compromiso y afecto que los lleva a permanecer e involucrarse en proyectos y metas para su propio desarrollo y mantenimiento.

Al intentar definir qué significa el "ser feriante" los integrantes señalan ser: "trabajadores y sacrificados", "buenos compañeros", "personas alegres y amables con el cliente" y por sobre todo "agraciados". Es posible dar cuenta que los individuos se consideran a sí mismos feriantes y reconocen las particularidades y diferencias que ello implica. Las características identificadas emergen del hecho de desempeñarse en una ocupación y contexto determinado, que considera formas y estilos de relaciones en función de su adaptación ocupacional.

En base a los resultados expuestos en este escrito, la ocupación, en este caso productiva, ha servido como forma a través de la cual se logra una adaptación constante a los diversos contextos sociopolíticos, vitales, temporales y espaciales de este grupo de individuos. Gracias a su cohesión en tanto miembros de un grupo particular que ha suscitado su participación en diversos roles con el objetivo común de perpetuarse como entidad diferenciada del común social. Esta forma de adaptarse implica la adopción de un estilo de vida imbricado con su principal ocupación productiva, la feria libre. Y su mantención en el tiempo se ha llevado a cabo gracias a las tradiciones y actividades que los caracterizan como tal es, como feriantes.

Por tanto nos atrevemos a afirmar que su principal ocupación productiva es en parte responsable de la conformación cultural del feriante, tanto en forma como en función y significado.

\section{Limitaciones y futuras investigaciones}

Por otro lado, en relación al proceso de investigación, las limitaciones y dificultades para la implementación y consecución de la misma, se encuentra el contexto sociocultural de los entrevistados y entrevistadores. La brecha existente entre ambos sobre la forma de expresión y comprensión del lenguaje, actuó como barrera para el entendimiento de formas un tanto abstractas como el sentido de pertenencia o significado que le atribuyen a su ocupación.

El rechazo hacia los interventores, de un determinado número de feriantes, se vio relacionado con la espera de una retribución tangible por parte del estudio en curso. Por otro lado, experiencias previas relacionadas a altas expectativas y promesas desde estudiantes de otras disciplinas que han realizado investigaciones con el grupo, ha generado molestia en parte de la población, negándose rotundamente a contribuir con la institución educativa. A esto se suma la relación que establecen con proyectos inconclusos de instituciones gubernamentales.

La falta de recursos teóricos y bibliográficos que brindaran información de las ferias libres en Chile, tanto en Terapia Ocupacional como en otras disciplinas académicas, se tornó un impedimento a la hora de investigar y buscar aproximaciones.

Dentro del proceso, como investigadores, se obtuvo información relevante para desarrollar la tesis planteada, sin embargo este nuevo conocimiento se generó de manera unidireccional, sin poseer instancias en donde se lograra contrastar, debatir y/o retroalimentar información de manera conjunta con la comunidad. Este fenómeno no fue posible llevarse a cabo, ya que al ser solicitado por la comunidad, el tiempo disponible para la investigación no fue suficiente para contener este importante segmento.

Finalmente, en relación a futuras investigaciones, se invita a miembros de la disciplina de la Terapia Ocupacional a contribuir a la investigación de la conformación de la ocupación en distintos contextos culturales, geográficos y/o sociopolíticos. 


\section{ReferenCias Bibliográficas}

Salazar, G. (2003). Ferias libres: espacio residual de la soberanía ciudadana (Reivindicación histórica) $\left(2^{\circ}\right.$ ed.). Santiago de Chile: McGraw-Hill

Confederación Gremial Nacional de Organizaciones de Ferias Libres, Persas y Afines. Extraído el 28 de septiembre 2012 desde http://www.asof.cl/cifras-ferias/.

Iwama, M. Kapanadze, M. \& Simó, S. (2008), En busca de una terapia ocupacional culturalmente relevante. Extraído de http:// www.revistatog.com/num8/pdfs/maestros.pdf.

Agostini, R.; Drumond, C.; Zocchi N. (2011). "La Terapia Ocupacional y su relación con la cultura contemporánea". Revista Terapia Ocupacional Galicia. Extraído desdehttp://www.revistatog. com/num13/pdfs/original3.pdf.

Iwama, M.; Simó, S. (2008). "Aspectos de significado, cultura en Terapia Ocupacional". TOG 2008; 5(8): [23p.]. Extraído de: http:// www.revistatog.com/num8/pdfs/modelo1.pdf.

Guajardo.; A., Algado, S. (2010). Una Terapia Ocupacional basada en los derechos humanos. Revista Gallega de Terapia Ocupacional. Galicia, España. Extraído el 28 de septiembre 2012 desde http://dialnet.unirioja.es/servlet/articulo?codigo=3674566.

Hernández, R.; Fernández, C.; Baptista, P. (2006). “Metodología de la investigación" (4ta ed.) México: McGraw-Hill.

Martínez, M. (2006). La Investigación Cualitativa (Síntesis Conceptual). Revista del Instituto de Investigaciones Psicológicas, Vol. 9, 123-146.

Taylor, S.J.; Bogdan R. (1987). "Introducción a los métodos cualitativos de investigación: La búsqueda de significados". Editorial Paidós Básica, pp. 100-132. Extraído desde http://cvonline. uaeh.edu.mx/Cursos/Lic_virt/Mercadotecnia/IMMC208/Unidad\%204/44_lec_La\%20entrevista\%20a\%20profundidad.pdf.

Olabuénaga, J.I. (2012). Metodología de la investigación cualitativa. (5ta ed.) Deusto: España.

Harris, M. (2000). Teorías sobre la Cultura en la era Posmoderna. (2da ed.) Barcelona: Crítica.

Iwama, M.; Kapanadze, M. \& Simó, S. (2008). En busca de una terapia ocupacional culturalmente relevante. Extraído desde http:// www.revistatog.com/num8/pdfs/maestros.pdf.

Iwama, M., Simó, S. "El Modelo Kawa" (Rio). TOG 2008; 5(8): [24 p.]. Extraído desde http://www.revistatog.com/num8/pdfs/modelo2.pdf.

Simó, S. (2012). "Terapia Ocupacional eco-social: hacia una ecología ocupacional". Extraído desde http://www.sos-ecosocial.org/ $\mathrm{pdf} / 4 / 25 . \mathrm{pdf}$.

Tappan, J. (1992). Cultura e identidad. Seminario sobre identidad: México: Instituto de Investigaciones Antropológicas, Universidad Nacional Autónoma de México.
Piqueras, A. (1996). El proceso deconstrucción de la identidad colectiva. (s.ed). Editorial Institució Valenciana D'estudios I investigació. Madrid: España.

García C. y Montero M. (2005). Una tendencia para pensar e investigar. Experiencia de investigación con una comunidad indígena Wayuu. Trabajo Social No. 7 (2005), páginas 173-190. Revista del Departamento de Trabajo Social, Facultad de Ciencias Humanas, Universidad Nacional de Colombia.

Cohen, A. (1986). Belonging identity and social organization in British rural cultures (s.ed.) Inglaterra: Manchester University Press.

Willard, H.; Spackman, C.; Crepeau, E.; Cohn, E.; Shell, B. (2010) Terapia Ocupacional: Willard and Spackman. 10a edición. Unidad I: Terapia Ocupacional y ciencia ocupacional: pasado, presente y futuro. Capítulo 2. Pág. 15-27. Unidad Madrid: Panamericana.

Redon, S. (2010). La escuela como espacio de ciudadanía. Escuela de Pedagogía Facultad de Filosofía y Educación - Pontificia Universidad Católica de Valparaíso, Chile). 